\title{
Review \\ Cognitive and neural plasticity in aging: General and task-specific limitations
}

\author{
Sari Jones ${ }^{\mathrm{a}, *}$, Lars Nyberg ${ }^{\mathrm{b}}$, Johan Sandblom ${ }^{\mathrm{c}}$, Anna Stigsdotter Neely ${ }^{\mathrm{b}}$, Martin Ingvar ${ }^{\mathrm{c}}$, \\ Karl Magnus Petersson ${ }^{\mathrm{c}, \mathrm{d}}$, Lars Bäckman ${ }^{\mathrm{a}, \mathrm{e}}$ \\ ${ }^{a}$ Aging Research Center, Division of Geriatric Epidemiology, Neurotec, Karolinska Institutet, Stockholm, Sweden \\ ${ }^{\mathrm{b}}$ Department of Psychology, Umeå University, Umeå, Sweden \\ ${ }^{c}$ Cognitive Neurophysiology Research Group R2-01, Department of Clinical Neuroscience, Karolinska Institutet, Stockholm, Sweden \\ ${ }^{\mathrm{d} C o g n i t i v e ~ N e u r o l o g y ~ a n d ~ M e m o r y ~ R e s e a r c h ~ G r o u p, ~ F . ~ C . ~ D o n d e r s ~ C e n t e r ~ f o r ~ C o g n i t i v e ~ N e u r o i m a g i n g, ~ M i j m e g e n, ~ T h e ~ N e t h e r l a n d s ~}$ \\ ${ }^{\mathrm{e}}$ Max Planck Institute for Human Development, Center for Lifespan Psychology, Berlin, Germany
}

\begin{abstract}
There is evidence for cognitive as well as neural plasticity across the adult life span, although aging is associated with certain constraints on plasticity. In the current paper, we argue that the age-related reduction in cognitive plasticity may be due to (a) deficits in general processing resources, and (b) failure to engage in task-relevant cognitive operations. Memory-training research suggests that agerelated processing deficits (e.g., executive functions, speed) hinder older adults from utilizing mnemonic techniques as efficiently as the young, and that this age difference is reflected by diminished frontal activity during mnemonic use. Additional constraints on memory plasticity in old age are related to difficulties that are specific to the task, such as creating visual images, as well as in binding together the information to be remembered. These deficiencies are paralleled by reduced activity in occipito-parietal and medial-temporal regions, respectively. Future attempts to optimize intervention-related gains in old age should consider targeting both general processing and task-specific origins of age-associated reductions in cognitive plasticity.
\end{abstract}

(C) 2006 Elsevier Ltd. All rights reserved.

Keywords: Cognitive aging; Plasticity; Memory training; Processing deficit; Utilization deficit

\section{Contents}

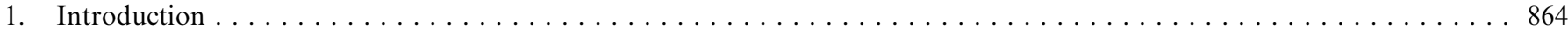

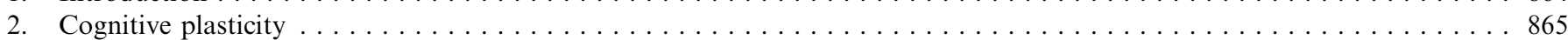

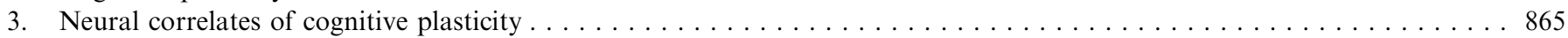

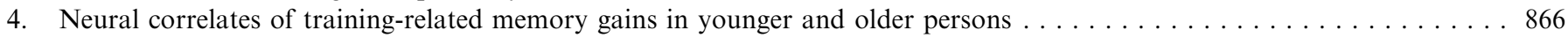

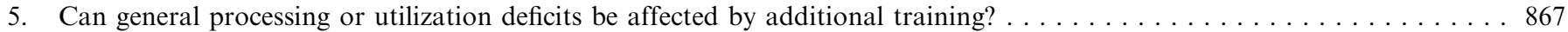

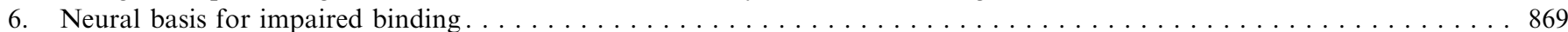

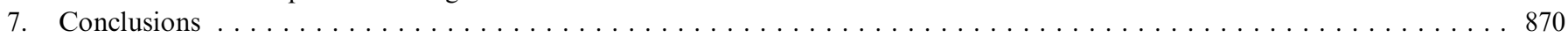

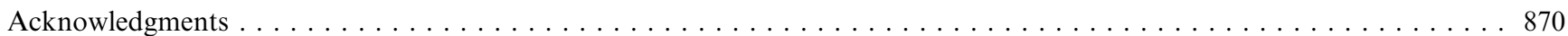

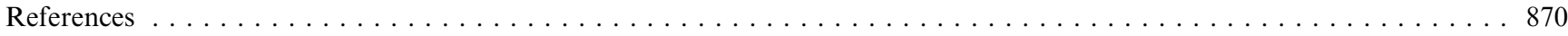

${ }^{*}$ Corresponding author. Tel.: + 4686906886 ; fax: +4686906889.

E-mail address: Sari.jones@neurotec.ki.se (S. Jones).

\section{Introduction}

It is well established that several areas of cognitive functioning (e.g., mental speed, episodic memory, working 
memory, fluid intelligence) decline with advancing adult age (Craik and Salthouse, 1999). This fact has spurred the interest for investigating ways of enhancing cognitive performance in old age, by improving cognitive abilities, or increasing the potential to use available cognitive resources, often referred to as plasticity. The aim of this review is twofold: (1) to highlight the potential for cognitive as well as neural plasticity in old age, focusing mainly on memory training, and (2) to provide possible explanations for the age-related reduction in memory plasticity, focusing on limitations in general processing and utilization factors.

\section{Cognitive plasticity}

Cognitive plasticity is a multifaceted concept that, among other things, denotes the potential to improve performance following training. Several studies have demonstrated training-related gains in memory using a variety of different mnemonic techniques (see Verhaegen et al., 1992; Stigsdotter Neely, 1994; Hill et al., 2000 for overviews). These techniques include, but are not limited to, the method of loci (MoL), the name-face mnemonic, and the pegword mnemonic. The MoL (Bower, 1970), which is a mnemonic technique for encoding words in serial order, is one of the most commonly used procedures in memory-training research. This method involves learning a series of landmarks (e.g., places at home, places along the way to work) in a specified order. When the loci have been acquired, words or other information are linked to the various landmarks at the time of encoding. At subsequent retrieval, the landmarks are mentally revisited and the words linked to each locus are retrieved in serial order.

A robust finding in this literature is that both younger and older adults show improvement following memory training as compared to placebo treatment or mere retesting. A few studies have shown that the memory training effects are reasonably well maintained over time periods up to three years (Stigsdotter Neely and Bäckman, 1993; Anschutz et al., 1987; Ball et al., 2002). Another finding to note is that no one strategy appears to be generally superior to others. Consistent with this observation, mnemonic strategies are quite specific in their applicability. For example, learning the face-name mnemonic is not very useful for remembering words in serial order. Hence, in memory-training studies, transfer effects to tasks not specifically trained are typically small or nonexistent (Stigsdotter Neely and Bäckman, 1993; Ball et al., 2002; Derwinger et al., 2003).

Further, a striking finding is that, although older persons benefit from memory training, younger adults benefit even more. Providing older persons with more extensive practice does not eliminate this difference (Baltes and Kliegl, 1992). Rather, differences in memory performance between younger and older persons tend to be magnified after extended training (Kliegl et al., 1989, 1990). This apparent age-related reduction in memory plasticity has also been observed within samples of older persons, such that the young-old exhibit larger training-related gains than the old-old (Singer et al., 2003). Hence, older age, is associated with constraints in memory plasticity. In addition, individual-difference variables (e.g. level of education, depression and dementia) are known to influence cognitive plasticity (Hill and Bäckman, 2000). Although disparate in nature, a common denominator of these variables is that they influence initial level of performance and in turn the magnitude of training-related gains.

To elucidate the mechanisms behind memory plasticity, Verhaegen and Marcoen (1996) tested older and younger persons on the MoL. They found several interesting correlates to the observed age differences in trainingrelated gains. One of these was that older participants failed to comply with the instructions. With shorter time for encoding ( $2 \mathrm{~min}$ for a list of 25 words) approximately $22 \%$ of the older individuals did not comply, and with longer time for encoding ( $6 \mathrm{~min})$, this figure dropped to about $5 \%$. By contrast, all younger participants applied the method consistently irrespective of task pacing. In addition, about $40 \%$ of the younger and about $66 \%$ of the older participants who complied with the mnemonic method, did not do so correctly; rather, they mixed the method with some other strategy, such as grouping related words or making up stories.

Finally, several cognitive abilities that are impaired in aging were related to the magnitude of training gains. Of these, associative memory had the strongest influence, followed by number of list rehearsals, and speed of processing. The results from this study suggest that differences in the size of training gains between younger and older persons may be due to age-related reductions in processing resources (a processing deficit), but also reflect the fact that older persons find it harder than younger persons to apply mnemonics in an appropriate manner (a utilization deficit).

To be sure, the fact that we differentiate between general and more specific constraints on plasticity in this article, should not be taken to mean that the two sets of factors are independent. Rather, it is highly conceivable that reduced processing-resources may lead to deficits in applying a mnemonic, especially if it relies heavily on workingmemory capacity.

\section{Neural correlates of cognitive plasticity}

Few studies have investigated the neural underpinnings of training-related memory enhancement. However, there is evidence of both functional and structural brain changes related to training of certain skills in young adults. Although only one of these studies targeted episodic memory, they all provide evidence of training-related neural plasticity.

Olesen et al. (2004) explored neural correlates of working-memory improvement using fMRI. Subjects who engaged in extensive practice in different working-memory 
tasks over five weeks improved their performance both on the tasks trained and on other unpracticed workingmemory tasks. This improvement was accompanied by increased brain activity in the left middle frontal gyrus and bilaterally in the inferior and superior parietal cortices.

New learning has also been associated with structural brain changes. Draganski and colleagues (2004) used whole-brain MRI to demonstrate an increase in grey matter in persons who were novices at juggling, but learnt the skill over a period of three months. Specifically, at posttest the jugglers showed bilateral expansion of grey matter in the mid-temporal area and in the left intraparietal sulcus. Interestingly, this expansion decreased after a further three-month interval during which no training occurred, although the volumetric estimates did not return to baseline levels. The latter result parallels findings from the animal literature indicating that neocortical neurogenesis may be transient (Gould et al., 2001). Although the locus of the effects observed by Draganski and colleagues remains unclear (e.g., synaptic density, neurites, glia cells, neurons), the findings indicate that neural alterations following training are not restricted to functional parameters (e.g., blood flow), and that the latter changes may have a structural basis.

Studies targeting the potential for older persons to exert neural plasticity are scarce. However, in one study magnetic resonance spectroscopy was used to measure biochemical changes in healthy older persons (mean age $=70$ years) after five weeks of training in the MoL (Valenzuela et al., 2003). Contrasting post-training with pre-training conditions revealed an elevation of creatine and choline signals in the hippocampus, but not in frontal or occipito-parietal regions. The selective changes observed may reflect an upregulation of oxidative metabolism in the form of increased neuronal energy available for synaptic transmission. The conclusions from the study were that these biochemical changes are persistent and may have a possible neuroprotective value in terms of extending the brain reserve in old age.

To the best of our knowledge, only one study has included both older and younger persons in investigating neural correlates of memory plasticity. This study will be reviewed in the next section.

\section{Neural correlates of training-related memory gains in younger and older persons}

In a PET study (Nyberg et al., 2003), we investigated brain activation in younger and older adults during encoding of novel words using the MoL. Eight younger and 16 older adults were scanned during pre-test, loci acquisition, and loci utilization. On the basis of test performance (see Table 1), the older sample was divided into two groups: those who benefited from the mnemonic (facilitated old or Old + ), and those who did not (unimproved old or Old-). At pre-test, participants memorized two separate lists of 18 words in serial order.
In the loci-acquisition part, they learnt 18 loci in a home (e.g., bed, cupboard, sofa) in a set order. All participants practiced acquisition until they managed to remember the loci serially during two consecutive trials with a maximum of one error. Hence, all participants learnt the mnemonic structure to perfection, although the unimproved old had a somewhat slower acquisition rate than the other groups. In the loci-utilization phase participants were instructed to use the mnemonic for remembering words from two separate lists. The behavioral data indicated that the young adults performed better than the older participants at pre-test, and also benefited more from using the mnemonic than the old, which is consistent with research reviewed in a previous section (Baltes and Kliegl, 1992; Kliegl et al., 1989, 1990). On average, the young improved by 5.12 words, the facilitated old by 3.75 words, and the unimproved old were actually worse off using the mnemonic. The latter group remembered, on average, more than two words fewer at post-test compared to pretest. Note that the two older groups performed at high levels and were indistinguishable on several cognitive ability tests (Table 1).

Consistent with the pattern of group differences at the behavioral level, there were also differences at the neural level among the young, the facilitated old, and the unimproved old when loci utilization was compared to pre-test (see Fig. 1). When contrasting post- and pre-test, the younger participants showed increased activity in left dorsolateral prefrontal cortex (DLPFC) during loci utilization. This finding was replicated in a recent fMRI study by Kondo et al. (2005), in which increased DLPFC activity was observed during utilization of the MoL mnemonic. Critically, however, increased DLPFC activity was not observed for either of the older groups in our PET study. In addition, the young and the facilitated old showed increased left occipito-parietal activity. The unimproved old showed no increased activity in either of these brain areas.

The lack of increase in activity in the DLPFC during loci utilization seen in both older groups may reflect an agerelated reduction in general processing capacity. Behaviorally, this was observed as lower scores on the digit symbol test, which assesses perceptual speed. These data converge with findings from Verhaegen and Marcoen (1996), who demonstrated an association between age-related reductions in training-related gains and different measures of processing resources. Findings from related studies show that slower processing speed is related to activity reductions (Rypma and D'Esposito, 2000) as well as volume losses (Raz et al., 1999) in the DLPFC.

The absence of activity in posterior occipito-parietal areas in the unimproved old was interpreted as a failure to engage in task-relevant operations. Specifically, activity in this area is related to visual-imagery processes (Cabeza and Nyberg, 2000; Kosslyn et al., 1996). Thus, although the unimproved old managed to learn the structure of the mnemonic, they seem to have problems associating visually 
Table 1

Subject characteristics and test performance (Adapted from Nyberg et al., 2003)

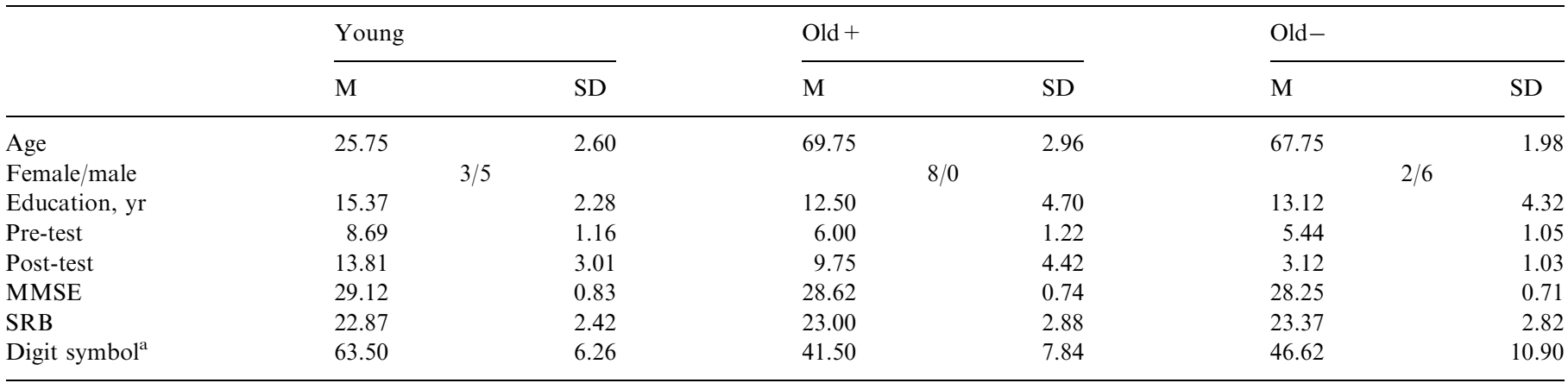

MMSE, Mini mental state examination (maximum score $=30$ ); SRB, Swedish vocabulary test (maximum score 30).

${ }^{\mathrm{a}}$ From the Wechsler adult intelligence scale revised (maximum score $=93$ ).
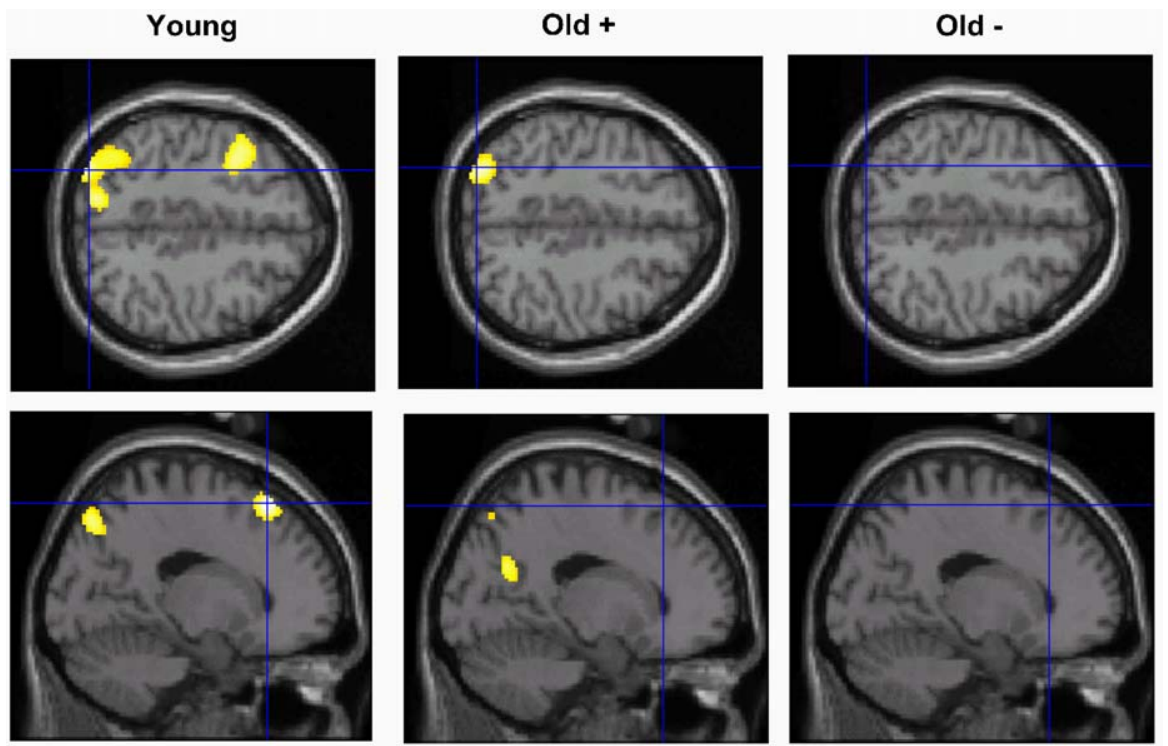

Fig. 1. Brain regions showing increased activity during loci utilization relative to pre-test in the three groups. The young and the facilitated old activated the occipitoparietal cortex. Only the young activated the left dorsal frontal cortex. (Adapted from Nyberg et al., 2003).

the target information with the loci compared to the two groups that showed training-related enhancement. Conceivably, this deficiency was reflected by lower activity in the occipito-parietal region. Hence, also at a neural level, age-related reductions in memory plasticity could be linked to both general processing and task-specific utilization factors.

\section{Can general processing or utilization deficits be affected by additional training?}

To further address the issue of how general processing and utilization factors impact on the potential for memory plasticity, all participants from the study reviewed above were invited back for follow-up cognitive tests, approximately one year later $(M=1.33, \mathrm{SD}=.29)$. All, except for one older person who had moved from the area, agreed to participate. The follow-up testing had two main aims: (1) to investigate whether the unimproved old are able to successfully utilize the MoL with additional training and more time for encoding; and (2) to explore the cognitive profiles among the groups with regard to tests assessing reasoning, executive/frontal functioning, visuospatial ability, and associative episodic memory. The latter objective was motivated by a desire to differentiate the facilitated and unimproved old cognitively; these two groups were indistinguishable on tasks of perceptual speed (Digit Symbol), verbal knowledge (SRB), and general cognitive ability (MMSE) in the main study (Table 1).

In the follow-up assessment, participants were first asked to relearn the same loci structure used in the original study, 18 words denoting places in a home. All learnt the loci structure to the same criterion as before and, as in the original study, the unimproved old needed more trials to reach the criterion (see Table 2). Having re-learnt the structure, participants were instructed to utilize the loci to encode 4 separate lists of new words. Immediately after encoding of each list, they were asked to recall as many 
Table 2

Test performance at follow-up

\begin{tabular}{|c|c|c|c|c|c|c|}
\hline & \multicolumn{2}{|c|}{ Young } & \multicolumn{2}{|c|}{ Old +} & \multicolumn{2}{|c|}{ Old- } \\
\hline & M & $\mathrm{SD}$ & M & SD & M & SD \\
\hline$N$ & \multicolumn{2}{|c|}{8} & \multicolumn{2}{|c|}{8} & \multicolumn{2}{|c|}{7} \\
\hline Training trials - loci structure & 3.50 & 1.07 & 4.87 & .83 & 6.14 & 1.35 \\
\hline Loci-overall & 14.37 & 2.60 & 8.41 & 3.85 & 5.07 & 1.97 \\
\hline Loci-fast & 14.31 & 2.74 & 7.69 & 3.93 & 4.36 & 2.30 \\
\hline Loci-slow & 14.43 & 2.97 & 9.12 & 4.43 & 5.79 & 2.14 \\
\hline
\end{tabular}

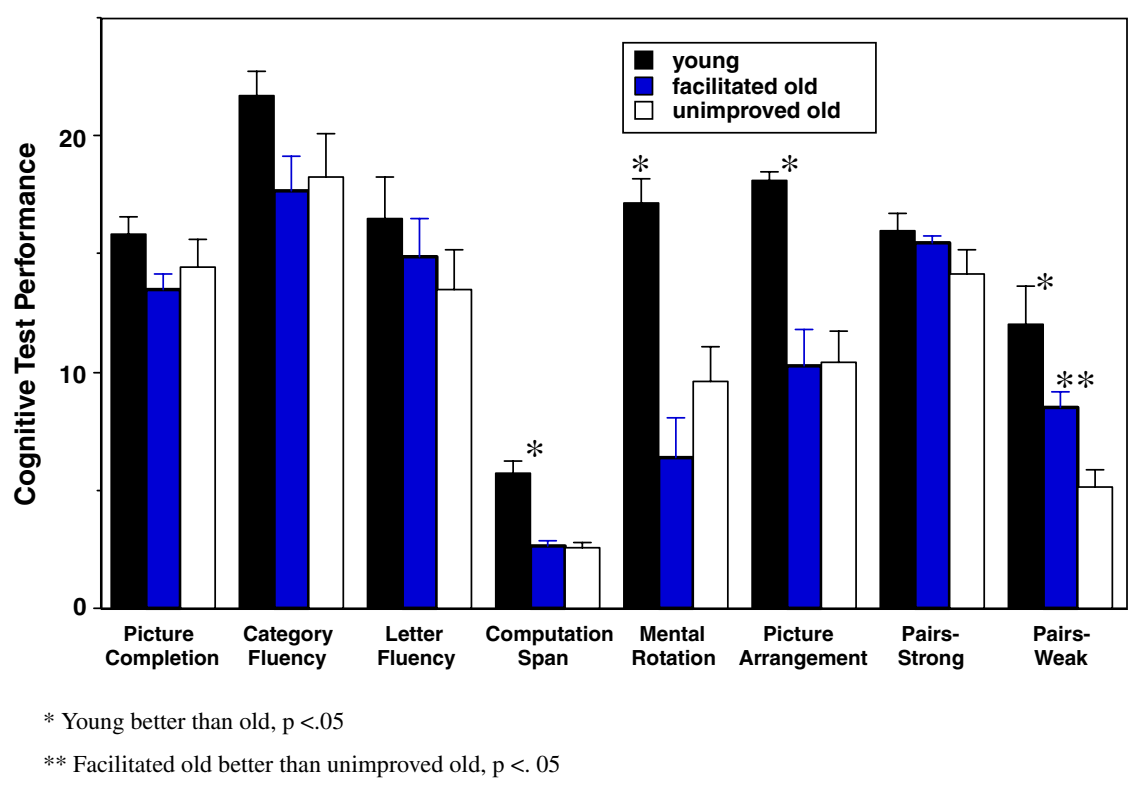

Fig. 2. Follow-up cognitive performance across the three groups.

words as possible in serial order. In two of the lists, words were presented for $5 \mathrm{~s}$ (fast) and in the other two for $10 \mathrm{~s}$ (slow). Table 2 presents overall memory performance as well as performance for the fast and slow encoding conditions.

Despite additional training, age differences in memory performance remained, which is in line with previous observations (Verhaegen et al., 1992). The young outperformed the facilitated old $(p<.01)$, who outperformed the unimproved old $(p<.10)$. More importantly, under identical pacing conditions, the unimproved old still performed worse after additional training (loci-fast, $M=4.4)$ than at pre-test $(M=5.4)$. Thus, the inability of the unimproved old to successfully utilize the MoL could not be alleviated by additional training. Structured interviews (e.g., "Could you clearly imagine the places that you had learnt?", "Could you associate the novel words with the places?", "Did you imagine walking through your home while recalling the words?"), after each loci-utilization task indicated that the inability was not due to limited understanding of how to use the mnemonic, but rather to a difficulty in implementing it. Further, more time for encoding resulted in performance gains of similar magni- tude for both older groups, but not to the extent that they were even close to the performance of the young.

The second aim of the follow-up study was to further investigate the cognitive profiles of the participants in order to delineate cognitive correlates of the group differences in the size of training-related gains. In the initial study, all three groups were comparable on global cognitive functioning and verbal knowledge, but as expected the young outperformed the older groups in digit symbol. In the follow-up study, tests of reasoning (picture completion, picture arrangement from the WAIS-R; Wechsler, 1981), executive/frontal functioning (category and letter fluency; Lezak, 1995; computation span; Salthouse and Babcock, 1991), visuospatial skill (mental rotations; Vandenberg, 1971) were administered according to standardized procedures.

In the initial study, some older participants expressed difficulty in forming associations between the loci and the words to be recalled. Therefore, we also included a pairedassociate learning task with weak and strong associates. In this task a list of 36 word pairs was prepared, using Swedish association norms (Shaps et al., 1976). The word pairs were presented in four random orders balanced 
across participants. At encoding, the word pairs were presented for $5 \mathrm{~s}$ each and participants were instructed to learn the pairs for later recall. Half of the pairs were strongly associated (e.g., arm-leg; table-chair) and half were weakly associated (e.g., rabbit-leaf; car-girl). At retrieval, participants were given the first words in the pairs as cues and were asked to retrieve the missing words. Also at retrieval, four random orders for word presentation were balanced across participants. The strong and weak word pairs were intermixed in the test, but were analyzed separately. Fig. 2 displays performance on the cognitive tests across the three groups.

The young participants performed better than both older groups on all tests. These findings are not surprising, given that the tests tax cognitive abilities known to deteriorate with advancing age. Statistically significant age-group differences were seen in picture arrangement, computation span, mental rotations, and paired-associates-weak. Conceivably, the lack of reliable age-related differences in the other tests partly reflects low statistical power. Given the imaging data from the original study, where only the young participants showed increased activation in frontal areas, behavioral differences in the tests assessing executive/ frontal functioning were indeed expected. The fact that no age-related differences were observed in the verbal fluency tasks may reflect the fact that these tasks draw on word knowledge in addition to their executive demands, the former being high in this sample of older individuals (see Table 1). The difference between younger and older adults in mental rotations may reflect the fact that older persons have a decreased ability to form and manipulate visual images (Dror and Kosslyn, 1994), which has been related to decreased frontal volume and low working memory capacity (Raz et al., 1999). Overall, the pattern of results in Fig. 2 provides further support for a general agerelated processing deficit.

The most interesting observation in this data set was that only paired-associates-weak discriminated between the facilitated old and the unimproved old. In pairedassociates-strong, all three groups performed close to ceiling, but when the associations were weak the young performed better than the facilitated old, who in turn outperformed the unimproved old, a similar pattern of group differences as in loci utilization. This intriguing finding encouraged us to perform some additional analyses of the original brain-imaging data.

\section{Neural basis for impaired binding}

One of the key characteristics of the MoL is that of information binding, that is, the formation of associations between novel words and different loci in a certain sequence. These associations can often turn out as unrealistic or even bizarre. Similarly, one of the key features of paired-associates-weak is to bind together two elements with weak pre-experimental resemblance. Associative learning and binding of information has been associated with increased activity in the medial temporal lobe (MTL) region (Cohen et al., 1999; Bunge et al., 2004; O'Reilly and Rudy, 2001). Therefore, we re-analyzed the imaging data to see whether areas in the MTL were differentially activated among the three groups during loci utilization. To achieve this objective, we conducted a directed search in the MTL region in which post-test (loci utilization) was contrasted against pre-test in the original data. The methods of image acquisition and data analysis were described in the original study (Nyberg et al., 2003) and will not be repeated here. However, for the new data presented here, pre-processing and analyses were done in SPM2 (www.fil.ion.ucl.ac.uk/spm).

This analysis revealed increased activity in the left MTL $(x, y, z=-18,-28,-24 ; p<0.05)$ for the young and the facilitated old, that is those who benefited from the mnemonic (Fig. 3). Interestingly, the young also showed increased activity in the right MTL $(x, y, z=28,-20,-24$; $p<0.05$ ). Hence, the young who performed best on pairedassociates-weak and benefited most from the MoL showed increased activity bilaterally in the MTL, the facilitated old who also benefited from the MoL, but not to the same degree as the young, showed increased activity in the left MTL only, whereas the unimproved old showed no increase of MTL activity. This pattern of data suggests a dose-response relationship between the degree of MTL activity, on the one hand, and successful use of the MoL and proficient binding of weakly associated word pairs, on the other.

An interesting contrast to these data is that in the original study (Nyberg et al., 2003) all participants showed
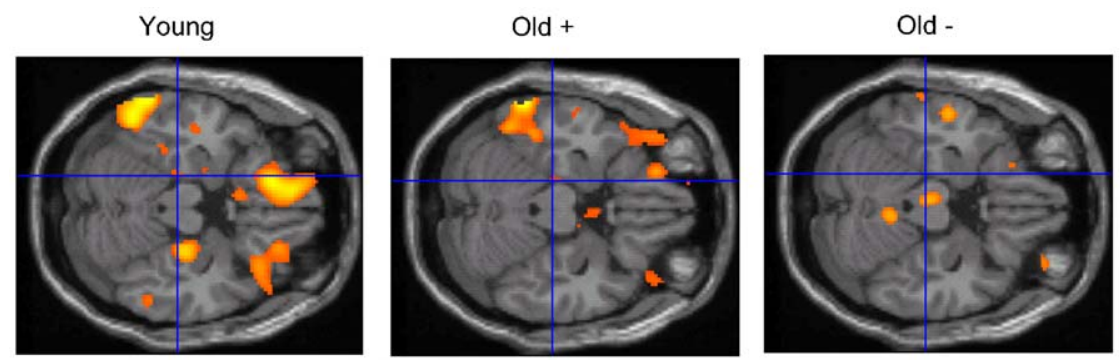

(Young \& Old+) - Old-

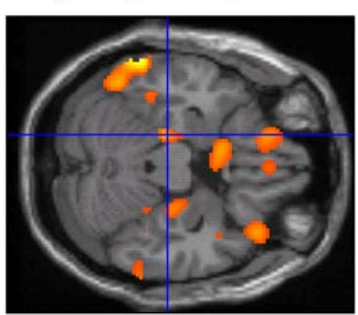

Fig. 3. Differential MTL activation in the three groups during loci utilization relative to pre-test. The young activated the left and the right MTL, the facilitated old activated the left MTL only, whereas the unimproved old showed no increase of MTL activity. 
increased hippocampal activity during loci acquisition. Learning the loci in serial order is clearly also dependent on binding of information; however, during loci acquisition the information has a coherent structure and the items (e.g., places at home) are highly related. Moreover, all participants successfully learnt the loci in serial order, which converges with the observation that they all showed increased MTL activity. Of further note in this context is that biochemical alterations have been demonstrated in the hippocampus (but not in frontal or occipito-parietal regions) after memory training with the MoL (Valenzuela et al., 2003). It is unclear, however, whether these alterations were specifically due to the memory-training task, or to increased mental activity in general.

The findings emerging from these additional analyses suggest that the utilization deficit of MoL in older adults may be linked to diminished activity in both occipitoparietal and MTL regions. Reduced activity in occipitoparietal regions may reflect an inability in visual-image processing, whereas the corresponding reduction of MTL activity may reflect a difficulty in information binding, both of which are important prerequisites for successful application of the MoL.

\section{Conclusions}

On the basis of past research and the original findings discussed in this article, we offer the following general conclusions: (1) Cognitive and neural plasticity are present across the adult life span, although there are age-related reductions in the potential for improvement; (2) There are consistent and cognitively plausible neural correlates of memory plasticity and age differences therein; and (3) Agerelated constraints in memory plasticity may reflect both a general processing (frontal) deficit, and a more task-specific utilization (parietal, MTL) deficit, having to do with visual imagery processing and binding together the critical elements when using the MoL.

With regard to the third conclusion, an interesting challenge for the future is to delineate which type of deficit (general processing or utilization) needs to be targeted for memory training with older adults to optimize performance gains. Training aimed specifically at task-specific processing deficits may involve finding the optimal strategy or technique to suit a particular individual, as clearly not all persons benefit from all techniques. Further, given the evidence for a general age-related processing deficit, it is of considerable interest to examine whether process-based (frontal-lobe) training will lead to enhanced cognitive performance in old age, as has been demonstrated with young adults (Olesen et al., 2004) as well as in children with cognitive deficits (Klingberg et al., 2002). If so, it will be of further interest to examine if training of one executive process (e.g., shifting) has beneficial effects on tests that tap other basic processes (e.g., interference resistance), or whether such gains are process-specific. If successful, this type of training is likely to benefit the majority of older persons. Further, it would be of interest to find out if process-based training could alleviate some of the agerelated utilization deficits in plasticity, as these likely interact.

\section{Acknowledgments}

This research was supported by grants from the Swedish Research Council and the Bank of Sweden Tercentenary Foundation to Lars Bäckman.

\section{References}

Anschutz, L., Camp, C.J., Markley, R.P., Kramer, J.J., 1987. Remembering mnemonics: a three-year follow-up on the effects of menmonics training in elderly adults. Experimental Aging Research 13, 141-143.

Ball, K., Berch, D.B., Helmers, K.F., Jobe, J.B., Leveck, M.D., Marsiske, M., Morris, J.N., Rebok, G.W., Smith, D.M., Tennstedt, S.L., Unversagt, F.W., Willis, S.L., 2002. Effects of cognitive training interventions with older adults. A randomized controlled trial. Journal of American Medical Association 288, 2271-2281.

Baltes, P.B., Kliegl, R., 1992. Further testing the limits of cognitive plasticity: Negative age differences in a mnemonic skill are robust. Developmental Psychology 28, 121-125.

Bower, G.H., 1970. Analysis of a mnemonic device. American Scientist 58, 496-510.

Bunge, S.A., Burrows, B., Wagner, A.D., 2004. Prefrontal and hippocampal contributions to visual associative recognition: interactions between cognitive control and episodic retrieval. Brain and Cognition $56,141-153$.

Cabeza, R., Nyberg, L., 2000. Imaging Cognition II: An empirical review of 275 PET and fMRI studies. Journal of Cognitive Neuroscience 12 (1), $1-47$.

Cohen, N.J., Ryan, J., Hunt, C., Romine, L., Wszalek, T., Nash, C., 1999. Hippocampal system and declarative (relational) memory: summarizing the data from functional neuroimaging studies. Hippocampus 9 , 83-98.

Craik, F.I.M., Salthouse, T.A. (Eds.), 1999. The Handbook of Aging and Cognition, vol. 2. Erlbaum, Mahwah, NJ.

Derwinger, A., Stigsdotter Neely, A., Persson, M., Hill, R.D., Bäckman, L., 2003. Remembering numbers in old age: mnemonic training versus self-generated strategy training. Aging, Neurospychology, and Cognition 10, 202-214.

Draganski, B., Gaser, C., Busch, V., Schuierert, G., Boghdan, U., May, A., 2004. Changes in grey matter induced by training. Nature 427 , 311-312.

Dror, I.E., Kosslyn, M., 1994. Mental imagery and aging. Psychology and Aging 9, 90-102.

Gould, E., Vail, N., Wagers, M., Gross, C.G., 2001. Adult-generated hippocampal and neocortical neurons in macaques have a transient existence. Proceedings of the National Academy of Sciences 98, 10910-10917.

Hill, R.D., Bäckman, L., 2000. Theoretical and methodological issues in memory training. In: Hill, R.D., Bäckman, L., Stigsdotter Neely, A. (Eds.), Cognitive rehabilitation in old age. Oxford University Press, New York, pp. 23-41.

Hill, R.D., Bäckman, L., Stigsdotter Neely, A. (Eds.), 2000. Cognitive Rehabilitation in Old Age. Oxford University Press, New York.

Kliegl, R., Smith, J., Baltes, P.B., 1989. Testing-the-limits and the study of adult age differences in cognitive plasticity of a mnemonic skill. Developmental Psychology 25, 247-256.

Kliegl, R., Smith, J., Baltes, P.B., 1990. On the locus and process of magnification of age differences during menmonic training. Developmental Psychology 26, 894-904. 
Klingberg, T., Forssberg, H., Westerberg, H., 2002. Training of working memory in children with ADHD. Journal of Clinical and Experimental Neuropsychology 24, 781-791.

Kondo, Y., Suzuki, M., Mugikura, S., Abe, N., Takahashi, S., Iijima, T., Fujii, T., 2005. Changes in brain activation associated with use of memory strategy: a functional MRI study. Neuroimage 24, 1154-1163.

Kosslyn, M., Thompson, W.L., Kim, I.J., Rauch, S.L., Alpert, N.M., 1996. Neural effects of visualizing and perceiving aversive stimuli: a PET investigation. Journal of Cognitive Neuroscience 8, 78-82.

Lezak, M.D., 1995. Neuropsychological Assessement, Third ed. Oxford University Press, New York.

Nyberg, L., Sandblom, J., Jones, S., Stigsdotter Neely, A., Petersson, K.M., Ingvar, M., Bäckman, L., 2003. Neural correlates of trainingrelated memory improvement in adulthood and aging. Proceedings of the National Academy of Sciences 100, 13728-13733.

Olesen, P.J., Westerberg, H., Klingberg, T., 2004. Increased prefrontal and parietal activity after training of working memory. Nature Neuroscience 7, 75-79.

O'Reilly, R.C., Rudy, J.W., 2001. Conjunctive representations in learning and memory: Principles of cortical and hippocampal function. Psychological Review 108, 311-345.

Raz, N., Briggs, S.D., Marks, W., Acker, J.D., 1999. Age-related deficits in generation and manipulation of mental images: The role of dorsolateral prefrontal cortex. Psychology and Aging 14, 436-444.

Rypma, B., D'Esposito, M., 2000. Isolating the neural mechanisms of agerelated changes in human working memory. Nature Neuroscience 3 , 509-515.
Salthouse, T.A., Babcock, R.L., 1991. Decomposing adult age differences in working memory. Developmental Psychology 27, 763-776.

Shaps, L., Johansson, B., Nilsson, L-G., 1976. Swedish association norms. Department of Psychology, Uppsala University, Uppsala, Sweden.

Singer, T., Lindenberger, U., Baltes, P.B., 2003. Plasticity of memory for new learning in very old age: a story of major loss? Psychology and Aging 18, 306-317.

Stigsdotter Neely, A., 1994. Memory training in late adulthood. Doctoral Dissertation, Department of Clinical Neuroscience and Family Medicine, Karolinska Institutet.

Stigsdotter Neely, A., Bäckman, L., 1993. Long-term maintenance of gains from memory training in older adults: Two $31 / 2$ years follow-up studies. Journals of Gerontology: Psychological Sciences 48, P233-P237.

Valenzuela, M.J., Jones, M., Wen, W., Rae, C., Graham, S., Shnier, R., Sachdev, P., 2003. Memory training alters hippocampal neurochemistry in healthy elderly. Neuroreport 14, 1333-1337.

Vandenberg, S.G., 1971. A test of three-dimensional spatial visualization based on the Shepard-Metzler mental rotation study. University of Colorado, Boulder.

Verhaegen, P., Marcoen, A., 1996. On the mechanisms of plasticity in young and older adults after instruction in the method of loci: evidence for an amplification model. Psychology and Aging 11, 164-178.

Verhaegen, P., Marcoen, A., Goossens, L., 1992. Improving memory performance in the aged through mnemonic training: a meta-analytic study. Psychology and Aging 7, 242-251.

Wechsler, D., 1981. Wechsler adult intelligence scale: revised. Psychological Corporation, New York. 